\title{
Correlation between minimum apparent diffusion coefficient values and the histological grade of breast invasive ductal carcinoma
}

\author{
SUHONG ZHAO ${ }^{1}$, WEIHUA GUO ${ }^{1}$, RU TAN ${ }^{2}$, PEIPEI CHEN ${ }^{1}$, ZHAOHUA LI ${ }^{1}$, \\ FENGGUO SUN $^{1}$ and GUANGRUI SHAO ${ }^{1}$ \\ ${ }^{1}$ Department of Radiology, The Second Hospital of Shandong University, Jinan, Shandong 250033; \\ ${ }^{2}$ Department of Radiology, Provincial Hospital of Shandong University, Jinan, Shandong 250021, P.R. China
}

Received April 20, 2017; Accepted December 6, 2018

DOI: $10.3892 / \mathrm{ol} .2018 .8343$

\begin{abstract}
The present study aimed to investigate the correlation between the minimum apparent diffusion coefficient $\left(\mathrm{ADC}_{\mathrm{min}}\right)$ value and the histological grade of breast invasive ductal carcinoma (IDC). In total, 129 pathologically verified lesions that were subjected to dynamic breast magnetic resonance imaging and diffusion weighted imaging prior to biopsy were included. The $\mathrm{ADC}_{\text {min }}$ value was calculated and its correlation with the tumor histological grade was investigated. Tumors of lower grades demonstrated significantly higher $\mathrm{ADC}_{\mathrm{min}}$ values as compared with tumors of higher grades $(\mathrm{F}=33.49 ; \mathrm{P}<0.01)$. The mean $\mathrm{ADC}_{\min }$ values for IDC of grades I, II and III were $(1.14 \pm 0.11) \times 10^{-3},(0.99 \pm 0.12) \times 10^{-3}$ and $(0.86 \pm 0.13) \times 10^{-3} \mathrm{~mm}^{2} / \mathrm{sec}$, respectively. Statistically significant differences were detected in the mean $\mathrm{ADC}_{\mathrm{min}}$ value between tumors of grades II and III $(\mathrm{P}<0.01)$, as well as between tumors of grades I and II $(\mathrm{P}<0.01)$. In addition, the mean $\mathrm{ADC}_{\min }$ values for the less aggressive (grades I and II) and more aggressive (grade III) groups were $(1.01 \pm 0.13) \times 10^{-3}$ and $(0.86 \pm 0.13) \times 10^{-3} \mathrm{~mm}^{2} / \mathrm{sec}$, respectively $(\mathrm{t}=5.76, \mathrm{P}<0.01)$. In conclusion, these data indicated that the $\mathrm{ADC}_{\text {min }}$ value was correlated with the IDC histological grade, and lower $\mathrm{ADC}_{\text {min }}$ values were associated with a higher histological grade and more aggressiveness. Thus, the $\mathrm{ADC}_{\text {min }}$ value may be considered as a promising prognostic parameter in identifying tumor aggressiveness.
\end{abstract}

\footnotetext{
Correspondence to: Dr Guangrui Shao, Department of Radiology, The Second Hospital of Shandong University, 247 Beiyuan Road, Jinan, Shandong 250033, P.R. China

E-mail: jn8193303@aliyun.com
}

Key words: invasive ductal carcinoma, histological grade, apparent diffusion coefficient

\section{Introduction}

Invasive ductal carcinoma (IDC), a heterogeneous disease, is the most common pathological type of breast cancer (1). There are three main prognostic determinants for breast cancer, including the lymph node status, tumor size and histological grade (2). The prognostic value of histological grade is considered to be equivalent to that of the lymph node status (3), whereas it is greater than that of tumor size (4). According to the World Health Organization (5), IDC can be classified into grades I, II and III, depending on the nuclear features, tubular formation and mitotic count. Compared with grades I and II, IDC of grade III is associated with a reduced time to relapse or mortality due to breast cancer (6). Therefore, the accurate determination of the IDC histological grade is particularly important for selection of the appropriate treatment and prediction of the disease prognosis.

Diffusion weighted imaging (DWI) is an advanced functional magnetic resonance imaging (MRI) technique, which is based on the measurement of water molecule diffusion in tissues $(7,8)$. Diffusion is quantified by the apparent diffusion coefficient (ADC), with low ADC values suggesting restricted diffusion (8). DWI has been initially applied for the diagnosis of acute stroke in clinical practice (9). With the rapid development of MRI techniques over the past years, the clinical application of DWI has been greatly extended for the imaging of various tumors (7-10). DWI is particularly helpful in the evaluation of breast masses by providing information on tumor behavior (8).

There have been numerous studies regarding the application of DWI in the diagnosis of breast lesions, and in the differential diagnosis between benign and malignant breast tumors $(11,12)$. The association between DWI and the histological grade of IDC has also been reported, although differential findings have been observed due to the use of a different region of interest (ROI) and measurement methods (13-20). The majority of previous studies have applied the mean ADC value to indicate the pathological characteristics of tumors. However, the minimum $\mathrm{ADC}\left(\mathrm{ADC}_{\mathrm{min}}\right)$ value is considered to be able to reflect the most malignant portions of tumors (21). In particular, the $\mathrm{ADC}_{\text {min }}$ value would be helpful for the selection 
of treatment plans if information on the IDC histological grade was also provided.

In the present retrospective study, the aim was to investigate the correlation of the $\mathrm{ADC}_{\text {min }}$ value with the histological grade and the aggressiveness of breast IDC.

\section{Materials and methods}

Study patients. In total, 281 patients with suspected with breast cancer were examined by breast MRI at the Second Hospital of Shandong University, (Jinan, Shandong, China) between May 2013 and July 2016 were reviewed. Among these cases, 152 cases were excluded due to receiving chemotherapy or radiotherapy $(n=25)$, lack of surgical confirmation $(n=26)$, benign lesions $(n=76)$ or suffering from other types of malignant tumors $(n=25)$. The remaining 129 patients with pathologically-diagnosed invasive ductal carcinoma according to the World Health Organization classification of tumors of the breast (5), who were all females, aged between 27 to 72 years old (median age of 48 years), with a mean age of $47.42 \pm 10.26$ years, were included into the analysis. Following MRI examination, all lesions were pathologically verified by lumpectomy, mastectomy or biopsy at the Institute of Pathology at the Second Hospital of Shandong University (Jinan, China). In order to avoid misdiagnosis caused by tumor heterogeneity, the biopsy was performed under the guidance of MRI inspection. Prior written informed consent was obtained from each patient, and the study was approved by the ethics committee of the Second Hospital of Shandong University.

MRI examination. All breast MRI examinations were performed on a 3.0-T system (GE Discovery MR750; GE Healthcare, Milwaukee, WI, USA), with an eight-channel dedicated breast coil. Patients were in the prone position, and both breasts were imaged simultaneously. For premenopausal females, imaging was performed between day 7 and day 14 of the menstrual cycle. An MRI plain scan was performed with the axial T2-weighted single-shot fast spin echo sequence using a modified Dixon technique $\left(\mathrm{T}_{2}\right.$ IDEAL) for intravoxel fat-water separation, using the following parameters: Repetition time (TR), 2,500 msec; echo time (TE), $53.5 \mathrm{msec}$; slice thickness, $6 \mathrm{~mm}$; layer spacing, $1.0 \mathrm{~mm}$; field of view, $360 \times 360 \mathrm{~mm}$; matrix size, 320x192 pixels; and number of excitations (NEX), 3. Similarly, the parameters for the axial T1-weighted fast spin-echo were as follows: TR, $569 \mathrm{msec}$; TE, $15.6 \mathrm{msec}$; slice thickness, $6 \mathrm{~mm}$; layer spacing, $1.0 \mathrm{~mm}$; field of view, 360x360 mm; matrix size, 256x192; NEX, 4.

In order to obtain dynamic contrast-enhanced (DCE) MRI scans, a dynamic examination was performed using the axial T1-weighted 3D dynamic gradient echo fat sequence. The parameters for this examination were as follows: TR/TE, 3.9/1.7; flip angle, $5^{\circ}$; field of view, $360 \times 360 \mathrm{~mm}$; matrix size, 348x348; and slice thickness, $1.8 \mathrm{~mm}$. Subsequent to unenhanced acquisition, Gadodiamide $(0.2 \mathrm{mmol} / \mathrm{kg}$ body weight, GE Healthcare Life Sciences, Little Chalfont, UK) was intravenously injected at the rate of $2 \mathrm{ml} / \mathrm{sec}$, followed by $20 \mathrm{ml}$ saline flush. DCE image acquisition was initiated immediately after the saline injection. The sequence was repeated for seven times without time gaps, and each sequence lasted for $60 \mathrm{sec}$.
DWI was performed with an axial single-shot fat suppressed echo-planar diffusion weighted sequence (TR, 3,000 msec; TE, $49.5 \mathrm{msec}$; slice thickness, $6 \mathrm{~mm}$; layer spacing, $1.0 \mathrm{~mm}$; field of view, $360 \times 360 \mathrm{~mm}$; matrix size, $128 \times 96$; and NEX, 4). The diffusion-sensitizing gradient was applied along the $x, y$ and $z$ axes, while b-values of 0 and $800 \mathrm{sec} / \mathrm{mm}^{2}$ were used.

Image analysis. MRI scans were independently reviewed by two experienced radiologists at a workstation (Advantage Windows Workstation 4.6; GE Healthcare) in a blinded manner. For each case, the final decision was made only upon agreement between these two radiologists. ADC measurement was performed in the GE workstation software. A ROI with a mean size of $25.7 \mathrm{~mm}^{2}$ (ranging between 8.0 and $79.0 \mathrm{~mm}^{2}$ ) was placed on the highest-signal focal in the DWI images that corresponded to the lowest-signal area in the ADC maps. Subsequently, the ADC values were automatically calculated on the ADC maps. The ROI was smaller than the lesion size and was placed in the solid part of IDC, avoiding the necrotic and hemorrhagic regions. The ROI size of each lesion was consistent for multiple measurements, in which the lowest of three measurements was accepted as the minimum ADC $\left(\mathrm{ADC}_{\min }\right)$ value.

Histopathological analysis. The histological grade of the tumors was assessed using the Nottingham modification of the Bloom-Richardson system (22), considering the following three parameters: i) Tubular formation (1 point, tubular formation in $>75 \%$ of the tumor; 2 points, tubular formation in $10-75 \%$; and 3 points, tubular formation in $<10 \%$ ); ii) nuclear pleomorphism (1 point, nuclei with minimal variation in size and shape; 2 points, moderate nuclear variation; and 3 points, marked nuclear variation); and iii) mitotic count (1 point, $0-11$ mitotic counts; 2 points, $12-22$ mitotic counts; and 3 points, $>23$ mitotic counts), calculated using a light microscope (BX43; Olympus Corporation, Tokyo, Japan) at magnification of $x 40$ with a field diameter of $0.63 \mathrm{~mm}$ and a field area of $0.312 \mathrm{~mm}^{2}$. The final decision on the histological grade was established only upon agreement of the investigators (two pathologists). Scores of 3-5, 6-7 and 8-9 were considered to indicate histological grades I, II and III, respectively (22).

Statistical analysis. Data are expressed as the mean \pm standard deviation. SPSS software (version 18.0; SPSS, Inc., Chicago, IL, USA) was used for statistical analysis. Due to the fact that the minimum $\mathrm{ADC}_{\min }$ or maximum $\mathrm{ADC}_{\text {min }}$ represented a specific value for each patient, no statistical analysis could be performed on these data. In the present study, the mean $\mathrm{ADC}_{\text {min }}$ value was calculated by averaging the values of all the patients. The mean $\mathrm{ADC}_{\text {min }}$ value difference was compared with the histological grade using the one-way analysis of variance and least significant difference test. Independent sample (Student's t-test) and receiver operating characteristic (ROC) curve analysis were used to analyze the diagnostic value of the mean $\mathrm{ADC}_{\min }$ value in differentiating less and more aggressive IDC cases. The optimal cutoff point of the mean $\mathrm{ADC}_{\text {min }}$ value, and the corresponding sensitivity and specificity were determined. $\mathrm{P}<0.05$ was considered to indicate differences that were statistically significant. 


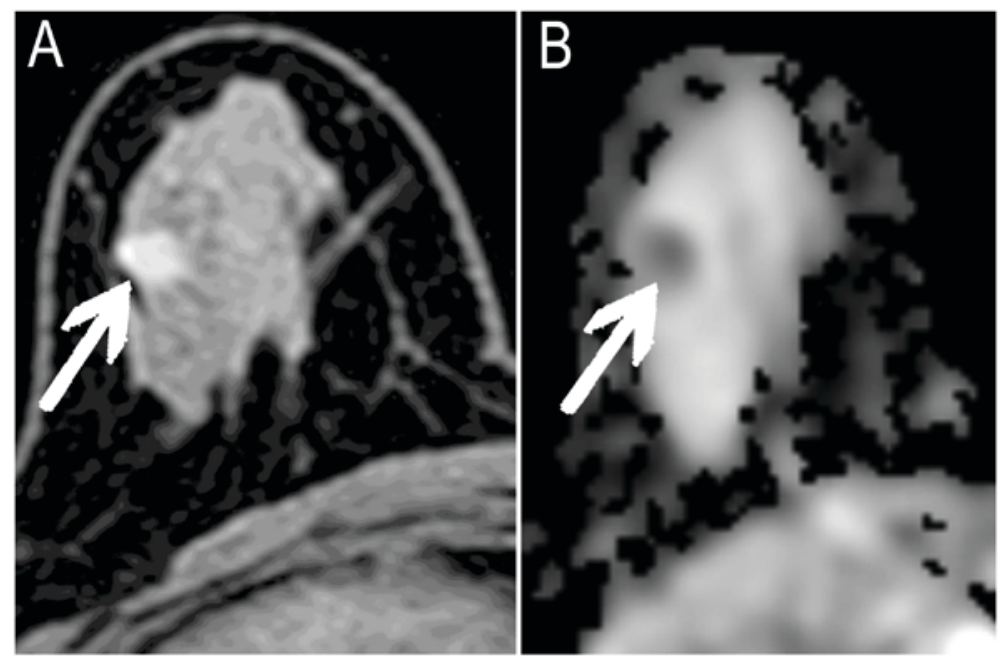

Figure 1. Representative imaging examination of histological grade I breast invasive ductal carcinoma in a 44-year-old female. (A) Axial contrast-enhanced image demonstrating the $1.2-\mathrm{cm}$ round mass with heterogeneous enhancement in the lower-outer quadrant of the right breast (arrow). (B) Axial ADC map of the breast demonstrating a low signal with a low $\mathrm{ADC}_{\min }$ value $\left(1.20 \times 10^{-3} \mathrm{~mm}^{2} / \mathrm{sec}\right)$ (arrow). $\mathrm{ADC}_{\min }$, minimum apparent diffusion coefficient.
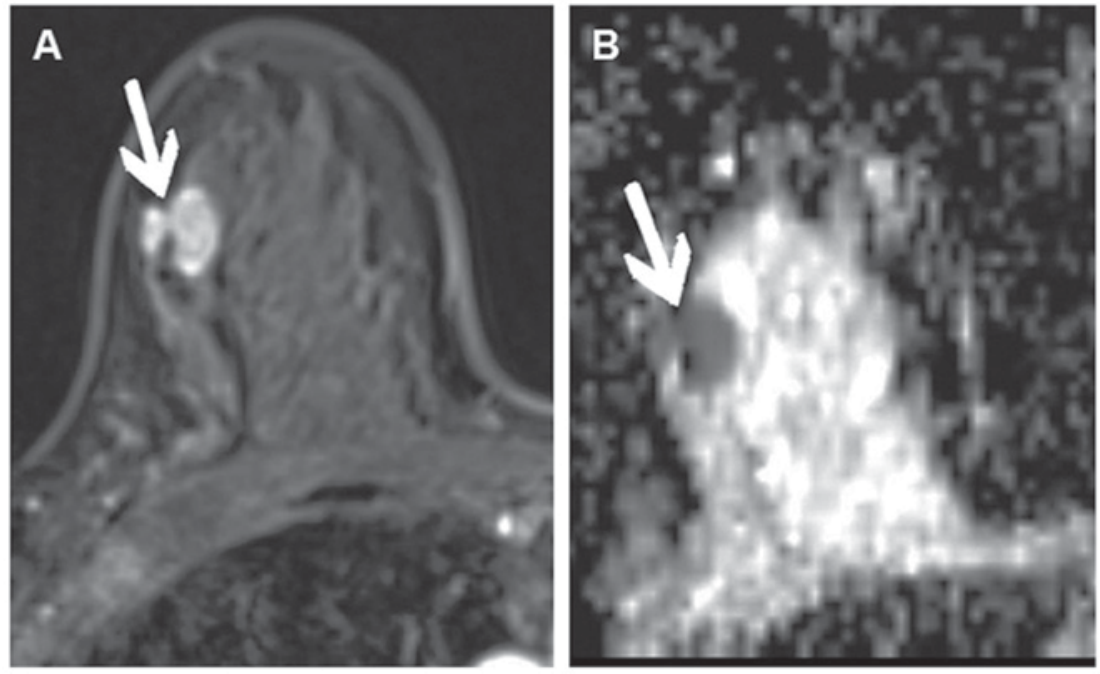

Figure 2. Representative imaging examination of histological grade II breast invasive ductal carcinoma in a 45-year-old female. (A) Axial contrast-enhanced image demonstrating the 1.3-cm irregular mass with heterogeneous enhancement in the upper-outer quadrant of the right breast (arrow). (B) Axial ADC map of the breast demonstrating a low signal with a low $\mathrm{ADC}_{\min }$ value $\left(0.96 \times 10^{-3} \mathrm{~mm}^{2} / \mathrm{sec}\right)$ (arrow). $\mathrm{ADC}_{\min }$, minimum apparent diffusion coefficient.

\section{Results}

Histological grade and $A D C_{\min }$ values in IDC patients. Among the 129 breast IDC cases included in the present study, pathological analysis revealed that there were 17 (13.18\%) cases of histological grade I, $79(61.24 \%)$ cases of histological grade II, and 33 (25.58\%) cases of histological grade III (Figs. 1-3). Furthermore, the mean $\mathrm{ADC}_{\text {min }}$ value of all IDC cases was $(0.97 \pm 0.15) \times 10^{-3} \mathrm{~mm}^{2} / \mathrm{sec}$. The minimum, maximum and mean $\mathrm{ADC}_{\min }$ values of IDC obtained by histological grade are presented in Table $\mathrm{I}$.

When considering the different histological grades of the included IDC patients, the mean $\mathrm{ADC}_{\min }$ values were $(1.14 \pm 0.11) \times 10^{-3},(0.99 \pm 0.12) \times 10^{-3}$ and $(0.86 \pm 0.13) \times 10^{-3} \mathrm{~mm}^{2} / \mathrm{sec}$ for patients with grade I, II and III disease, respectively. The corresponding ranges of these values were $(0.95-1.34) \times 10^{-3}$, $(0.72-1.30) \times 10^{-3}$ and $(0.50-1.10) \times 10^{-3} \mathrm{~mm}^{2} / \mathrm{sec}$, respectively.
These measurement results revealed that different $\mathrm{ADC}_{\mathrm{min}}$ values corresponded to IDC cases with different histological grades. Compared with cases of higher grades, tumors of lower grades exhibited significantly higher ADC values $(\mathrm{F}=33.49$; $\mathrm{P}<0.01)$. In particular, there was a significant difference in the $\mathrm{ADC}_{\min }$ value between grade II and III tumors $(\mathrm{P}<0.01)$, as well as between grade I and II tumors ( $\mathrm{P}<0.01$; Fig. 4). Taken together, these results suggest that, the $\mathrm{ADC}_{\min }$ value is inversely correlated with the histological grade of IDC.

$A D C_{\min }$ value and disease aggressiveness in IDC patients. To evaluate the role of the $\mathrm{ADC}_{\min }$ value in determining the aggressiveness of IDC, the cases were divided into the less aggressive (grades I and II) and more aggressive (grade III) groups. The results revealed that the mean $\mathrm{ADC}_{\min }$ values in the less aggressive group was $(1.01 \pm 0.13) \times 10^{-3} \mathrm{~mm}^{2} / \mathrm{sec}$, while this value was $(0.86 \pm 0.13) \times 10^{-3} \mathrm{~mm}^{2} / \mathrm{sec}$ in the more aggressive 

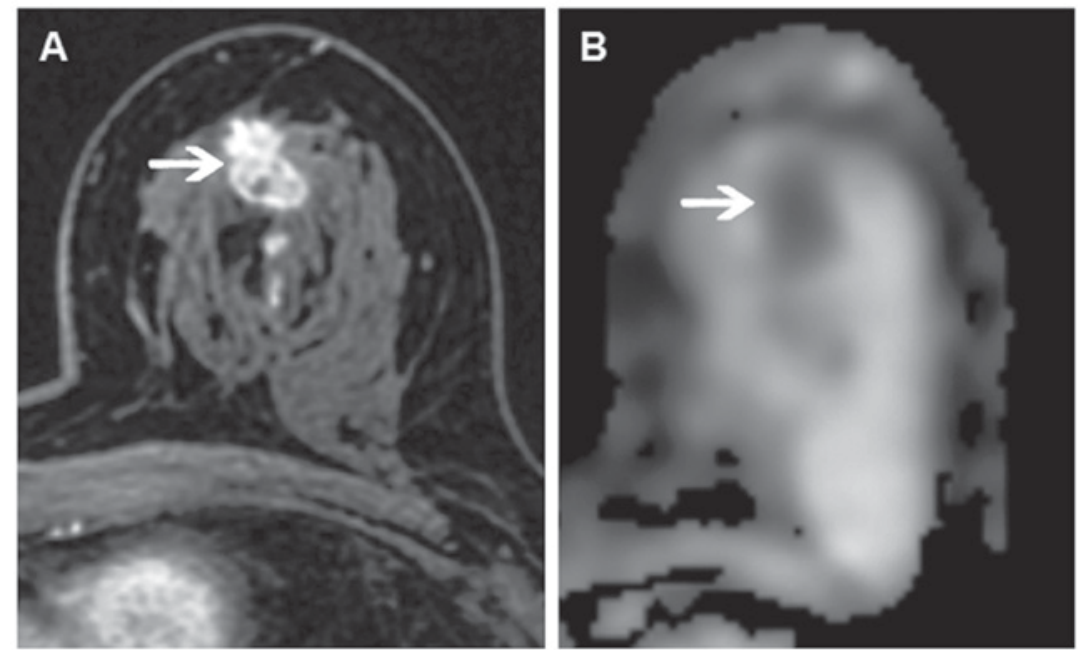

Figure 3. Representative imaging examination of histological grade III breast invasive ductal carcinoma in a 53-year-old female. (A) Axial contrast-enhanced image demonstrating the 1.6- $\mathrm{cm}$ irregular mass with heterogeneous enhancement in the central area of the left breast (arrow). (B) Axial ADC map of the breast demonstrating a low signal with a low $\mathrm{ADC}_{\min }$ value $\left(0.78 \times 10^{-3} \mathrm{~mm}^{2} / \mathrm{sec}\right)$ (arrow). $\mathrm{ADC}_{\min }$, minimum apparent diffusion coefficient.

Table I. Range and mean values of $\mathrm{ADC}_{\min }$ of IDC according to the different histological grades.

\begin{tabular}{lcccr}
\hline Grade & $\mathrm{n}$ & $\mathrm{ADC}_{\text {min }}$ range $\left(\mathrm{x} 10^{-3} \mathrm{~mm}^{2} / \mathrm{sec}\right)$ & $\mathrm{Mean}_{\mathrm{ADC}}$ min $\left(\times 10^{-3} \mathrm{~mm}^{2} / \mathrm{sec}\right)$ & $\mathrm{P}$-value \\
\hline I & 17 & $0.95-1.34$ & $1.14 \pm 0.11$ & $<0.01$ \\
II & 79 & $0.72-1.30$ & $0.99 \pm 0.12$ & $<0.01$ \\
III & 33 & $0.50-1.10$ & $0.86 \pm 0.13$ & $<0.01$ \\
\hline
\end{tabular}

P-values were obtained from comparison with the mean $\mathrm{ADC}_{\min }$ groups. $\mathrm{ADC}_{\min }$, minimum apparent diffusion coefficient.

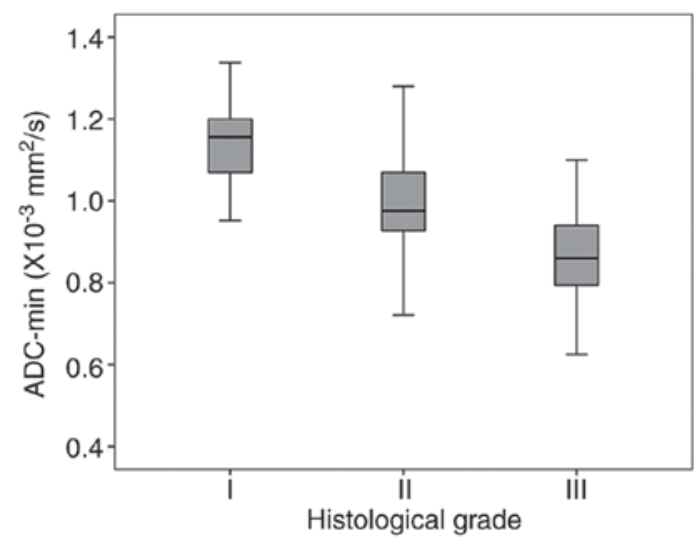

Figure 4. Box plot demonstrating the correlation between the $\mathrm{ADC}_{\min }$ value and the histological grade of IDC. Mean $\mathrm{ADC}_{\min }$ values for IDC cases of histological grades I, II and III were $(1.14 \pm 0.11) \times 10^{-3},(0.99 \pm 0.12) \times 10^{-3}$ and $(0.86 \pm 0.13) \times 10^{-3} \mathrm{~mm}^{2} / \mathrm{sec}$, respectively $(\mathrm{P}<0.01) . \mathrm{ADC}_{\min }$, minimum apparent diffusion coefficient; IDC, invasive ductal carcinoma.

group ( $\mathrm{t}=5.76, \mathrm{P}<0.01)$. These results suggest that the $\mathrm{ADC}_{\min }$ value is inversely correlated with the aggressiveness of IDC.

$R O C$ analysis of $A D C_{\min }$ value in IDC diagnosis. ROC analysis demonstrated that the $\mathrm{ADC}_{\min }$ value was a significant parameter in the diagnosis of less aggressive IDC, with an area under the curve (AUC) of 0.81 . The $\mathrm{ADC}_{\min }$ threshold value

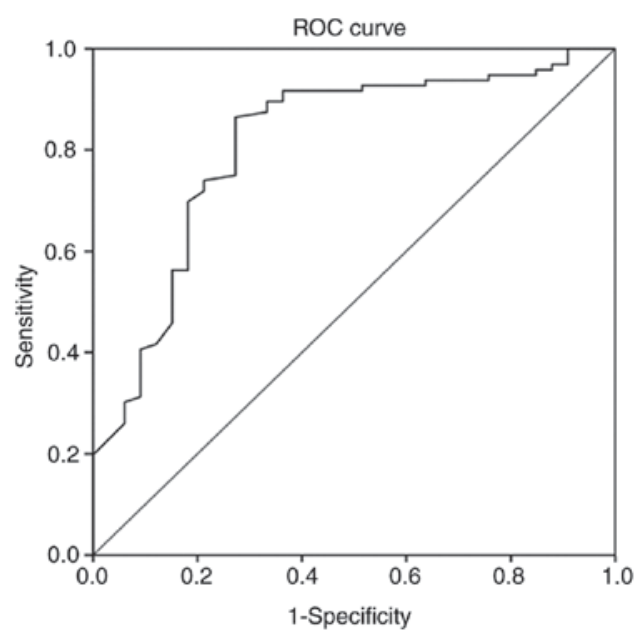

Figure 5. Receiver operating characteristic curve analysis of $\mathrm{ADC}_{\min }$ values in the diagnosis of IDC. The $\mathrm{ADC}_{\min }$ value exhibited great significance in the diagnosis of less aggressive IDC cases, with an area under the curve of 0.81 . The $\mathrm{ADC}_{\min }$ threshold value of $0.90 \times 10^{-3} \mathrm{~mm}^{2} / \mathrm{sec}$ corresponded to a sensitivity of $86.5 \%$ and a specificity of $72.7 \%$ for the detection of less aggressive tumors. $\mathrm{ADC}_{\min }$, minimum apparent diffusion coefficient; IDC, invasive ductal carcinoma.

of $0.90 \times 10^{-3} \mathrm{~mm}^{2} / \mathrm{sec}$ corresponded to a sensitivity of $86.5 \%$ and a specificity of $72.7 \%$ in the detection of less aggressive tumors (Fig. 5). These results suggest the importance of the $\mathrm{ADC}_{\min }$ value in diagnosing less aggressive IDC. 


\section{Discussion}

The histological grade represents an important prognostic factor for tumors in clinical practice, which is helpful in evaluating the tumor behavior (2). In addition, DWI is quick examination that does not require the use of a contrast agent and is quantified by the apparent diffusion coefficient. Moreever, the $\mathrm{ADC}$ value is quantitative and, therefore, an objective calculation $(8,23)$. Previous studies have examined the association between the ADC value and tumor grade. For instance, certain studies have demonstrated that the ADC value is inversely correlated with the tumor grade (13-15). Cipolla et al (16) also revealed that the ADC values were significantly higher in G1 tumors as compared with G3 tumors, while there was no statistically significant difference upon comparison of G1 and G3 tumors with G2 tumors. By contrast, several other studies have indicated that no correlation exists between the ADC value and tumor grade (17-20).

In the present study, the results demonstrated that the mean $\mathrm{ADC}_{\min }$ values for IDC of grades I, II and III were $(1.14 \pm 0.11) \times 10^{-3},(0.99 \pm 0.12) \times 10^{-3}$ and $(0.86 \pm 0.13)$ $\mathrm{x} 10^{-3} \mathrm{~mm}^{2} / \mathrm{sec}$, respectively, with the corresponding ranges of $(0.95-1.34) \times 10^{-3},(0.72-1.30) \times 10^{-3}$ and $(0.50-1.10) \times 10^{-3} \mathrm{~mm}^{2} / \mathrm{sec}$. Tumors of lower grades were observed to exhibit significantly higher ADC values compared with tumors of higher grades. In addition, there was significant difference in the $\mathrm{ADC}_{\text {min }}$ value between tumors of grades II and III, as well as between tumors of grades I and II. This phenomenon may be attributed to the higher cellular density and smaller extracellular space in IDC of higher grades, which results in lower ADC values (24). Additionally, the overlapping of the $\mathrm{ADC}_{\min }$ for the three grades of IDC is possibly due to tumor heterogeneity (1).

The majority of previous studies (13-20) have used the mean ADC value when investigating its correlation with the tumor grade. However, in the present study, the $\mathrm{ADC}_{\min }$ values were used instead, and significant differences were observed in these values between different tumor grades. IDC is a heterogeneous tumor consisting of invasive cancer nests, fibrosis and necrosis. In MRI scans, the regions of the maximum ADC $\left(\mathrm{ADC}_{\max }\right)$ values reflect the lowest cellular zone, while the regions of the $\mathrm{ADC}_{\min }$ values reflect the highest cellular zone composed of stroma (21). Furthermore, the presence of fibrosis and necrosis may affect the ADC values, particularly the $\mathrm{ADC}_{\max }$ values. Therefore, there will always be differences in the $\mathrm{ADC}_{\max }$ values in the local measurement and pathological characterization of tumors. In the present study, the $\mathrm{ADC}_{\min }$ value was used for tumor pathological characterization, which has been suggested to reflect the most malignant portions of tumors (21).

There have been several studies investigating the application of the $\mathrm{ADC}_{\min }$ values in differentiating the benign and malignant breast masses (as well as in differentiating breast cancer subtypes), and in detecting the invasive component in ductal carcinoma in situ $(21,25,26)$. Byun et al (27) have measured the mean $\mathrm{ADC}$ and the mean $\mathrm{ADC}_{\min }$ values in the regions with the highest fluorodeoxyglucose (FDG) uptake using sequential ${ }^{18} \mathrm{~F}-\mathrm{FDG}$ positron-emission tomography and MRI, and examined the correlation of the corresponding ADC values with the histological grade of IDC. The majority of these aforementioned studies have applied the multiple ROI method in breast MRI, and the $\mathrm{ADC}_{\min }$ value represented the lowest mean ADC value among multiple small ROIs within the lesion. However, this method is time-consuming, limiting its clinical application $(21,25,27)$. In the present study, the single ROI was smaller in size when compared with the lesion size, and was placed in the solid part of the IDC, avoiding the necrotic and hemorrhagic regions. The ROI size of each lesion was consistent for multiple measurements, and the lowest ADC value among three measurements was determined as the $\mathrm{ADC}_{\mathrm{min}}$ for each lesion. This method has been previously reported, with considerable feasibility in clinical settings (26). Furthermore, the results of the current study were consistent with previous findings (13-15), with a simpler and more feasible method used, and confirmed that the $\mathrm{ADC}_{\min }$ value was inversely correlated with the histological grade of breast IDC. Further studies with larger sample sizes are required to evaluate the clinical application of the $\mathrm{ADC}_{\text {min }}$ value in tumor grading.

Biological evaluation of tumors is important for the selection of treatment options. Different tumor cell densities may indicate different histological structures and biological invasions. The ADC values for IDC lesions were lower as compared with those of other malignant tumors, which may be due to the densely packed tumor cells, restricting effective motion and diffusion of water molecules (28). Costantini et al (13) have identified that the mean ADC value for IDC was $1.03 \times 10^{-3} \mathrm{~mm}^{2} / \mathrm{sec}$, while the mean ADC value for ductal carcinoma in situ was $1.05 \times 10^{-3} \mathrm{~mm}^{2} / \mathrm{sec}$. In the present study, the mean $\mathrm{ADC}_{\min }$ value for IDC was $(0.97 \pm 0.15) \times 10^{-3} \mathrm{~mm}^{2} / \mathrm{sec}$, while the values for the less aggressive (grades I and II) and more aggressive (grade III) tumor groups were $(1.01 \pm 0.13) \times 10^{-3}$ and $(0.86 \pm 0.13) \times 10^{-3} \mathrm{~mm}^{2} / \mathrm{sec}$, respectively. Thus, a significant difference was observed in the mean $\mathrm{ADC}_{\text {min }}$ value between the less and more aggressive IDC groups, which was in line with previous observations (13). Furthermore, a cutoff point for the $\mathrm{ADC}_{\min }$ threshold value of $0.90 \times 10^{-3} \mathrm{~mm}^{2} / \mathrm{sec}$ was used to detect the less aggressive tumors in the present study, corresponding to a sensitivity of $86.5 \%$ and a specificity of $72.7 \%$. The results of the ROC curve analysis further revealed that the $\mathrm{ADC}_{\min }$ value was important in the diagnosis of less aggressive IDC cases, with an AUC of 0.81 .

There are also certain limitations in the present study. Firstly, the sample size was relatively small; therefore, the findings need to be validated in studies using larger sample sizes. In addition, all lesions herein were IDCs, appearing as a mass-like enhancement. Therefore, it was not able to evaluate the $\mathrm{ADC}_{\min }$ value of other types of breast carcinoma appearing as non mass-like enhancement with respect to the pathological grade. Furthermore, the measurement of $\mathrm{ADC}_{\min }$ value was relatively subjective, which may lead to observational bias. Another limitation is that the association between the histological grade and the morphological alterations, as well as the enhancement pattern, were not examined in the present study. Routine imaging findings combined with the ADC value would improve the accuracy of the preoperative assessment of the histological grade for IDC, which would assist in the selection of the appropriate treatment options for breast cancer. Additionally, the association between the $\mathrm{ADC}_{\min }$ values and other pathological characteristics was not investigated herein, which should be considered in further in-depth studies in the 
future. According to a previous study (29) and clinical practice, only two b-values (0 and $800 \mathrm{~mm}^{2} / \mathrm{sec}$ ) were applied in the present study; thus, DWI images with more b values would be required in the future to obtain accurate ADC values. Finally, the present study was a retrospective study, and therefore, the $\mathrm{ADC}_{\min }$ value was not considered in the clinical decisions.

In conclusion, the results of the current study indicated that the $\mathrm{ADC}_{\text {min }}$ value was correlated with the histological grade of IDC. Lower $\mathrm{ADC}_{\text {min }}$ values were associated with higher histological grades. These findings suggest that the $\mathrm{ADC}_{\text {min }}$ value may be considered as a promising prognostic parameter in identifying tumor aggressiveness.

\section{Acknowledgements}

Not applicable.

\section{Funding}

This study was supported by the Key Research and Development Plan of Shandong Province (grant no. 2015GSF119034) and the Seed Fund of the Second Hospital of Shandong University (grand no. S2014010020).

\section{Availability of data and materials}

The datasets used and/or analyzed during the current study are available from the corresponding author on reasonable request.

\section{Authors' contributions}

GS and WG designed the study. SZ, RT, PC, ZL, and FS performed the experiments. SZ analysed the data.

\section{Ethics approval and consent to participate}

The present study was approved by the ethics committee of the Second Hospital of Shandong University.

\section{Consent for publication}

Prior written informed consent was obtained from each patient.

\section{Competing interests}

The authors declare that they have no competing interests.

\section{References}

1. Hawthorn L, Luce J, Stein L and Rothschild J: Integration of transcript expression, copy number and $\mathrm{LOH}$ analysis of infiltrating ductal carcinoma of the breast. BMC Cancer 10: 460, 2010.

2. Rakha EA, Reis-Filho JS, Baehner F, Dabbs DJ, Decker T, Eusebi V, Fox SB, Ichihara S, Jacquemier J, Lakhani SR, et al: Breast cancer prognostic classification in the molecular era: The role of histological grade. Breast Cancer Res 12: 207, 2010.

3. Wirapati P, Sotiriou C, Kunkel S, Farmer P, Pradervand S, Haibe-Kains B, Desmedt C, Ignatiadis M, Sengstag T, Schütz F, et al: Meta-analysis of gene expression profiles in breast cancer: Toward a unified understanding of breast cancer subtyping and prognosis signatures. Breast Cancer Res 10: R65, 2008 .
4. Sundquist M, Thorstenson S, Brudin L, Nordenskjold B and Nordenskjöld B: Applying the nottingham prognostic index to a Swedish breast cancer population. South East Swedish Breast Cancer Study Group. Breast Cancer Res Treat 53: 1-8, 1999.

5. Elston CW and Ellis IO: Pathological prognostic factors in breast cancer. I. The value of histological grade in breast cancer: Experience from a large study with long-term follow-up. Histopathology 19: 403-410, 1991.

6. Trudeau ME, Pritchard KI, Chapman JA, Hanna WM, Kahn HJ, Murray D, Sawka CA, Mobbs BG, Andrulis I, McCready DR and Lickley HL: Prognostic factors affecting the natural history of node-negative breast cancer. Breast Cancer Res Treat 89: 35-45, 2005.

7. Guo Y, Cai YQ, Cai ZL, Gao YG, An NY, Ma L, Mahankali S and Gao JH: Differentiation of clinically benign and malignant breast lesions using diffusion-weighted imaging. J Magn Reson Imaging 16: 172-178, 2002.

8. Bammer R: Basic principles of diffusion-weighted imaging. Eur J Radiol 45: 169-184, 2003.

9. Sinha S, Lucas-Quesada FA, Sinha U, DeBruhl N and Bassett LW: In vivo diffusion-weighted MRI of the breast: Potential for lesion characterization. J Magn Reson Imaging 15: 693-704, 2002.

10. Hosseinzadeh K and Schwarz SD: Endorectal diffusion-weighted imaging in prostate cancer to differentiate malignant and benign peripheral zone tissue. J Magn Reson Imaging 20: 654-661, 2004.

11. Zhao J, Guan H, Li M, Gu H, Qin J and Wu X: Significance of the ADC ratio in the differential diagnosis of breast lesions. Acta Radiol 57: 422-429, 2016

12. Çabuk G, Nass Puce M, Özgür A, Apaydın FD, Polat A and Orekici G: The diagnostic value of diffusion-weighted imaging and the apparent diffusion coefficient values in the differentiation of benign and malignant breast lesions. J Med Imaging Radiat Oncol 59: 141-148, 2015.

13. Costantini M, Belli P, Rinaldi P, Bufi E, Giardina G, Franceschini G, Petrone G and Bonomo L: Diffusion-weighted imaging in breast cancer: Relationship between apparent diffusion coefficient and tumour aggressiveness. Clin Radio 65: 1005-1012, 2010

14. Razek AA, Gaballa G, Denewer A and Nada N: Invasive ductal carcinoma: Correlation of apparent diffusion coefficient value with pathological prognostic factors. NMR Biomed 23: 619-623, 2010.

15. Belli P, Costantini M, Bufi E, Giardina GG, Rinaldi P, Franceschini G and Bonomo L: Diffusion magnetic resonance imaging in breast cancer characterization: Correlations between the apparent diffusion coefficient and major prognostic factors. Radiol Med 120: 268-276, 2015.

16. Cipolla V, Santucci D, Guerrieri D, Drudi FM, Meggiorini ML and de Felice C: Correlation between 3T apparent diffusion coefficient values and grading of invasive breast carcinoma. Eur J Radio 183: 2144-2150, 2014.

17. Park SH, Choi HY and Hahn SY: Correlations between apparent diffusion coefficient and invasive ductal carcinoma and pathologic factors on diffusion-weighted imaging MRI at 3.0 Tesla. J Magn Reson Imaging 41: 175-182, 2015.

18. Durando M, Gennaro L, Cho GY, Giri DD, Gnanasigamani MM, Patil S, Sutton EJ, Deasy JO, Morris EA and Thakur SB: Quantitative apparent diffusion coefficient measurement obtained by 3.0 Tesla MRI as a potential noninvasive marker of tumor aggressiveness in breast cancer. Eur J Radiol 85: 1651-1658, 2016.

19. Park EK, Cho KR, Seo BK, Woo OH, Cho SB and Bae JW: Additional value of diffusion-weighted imaging to evaluate prognostic factors of breast cancer: Correlation with the apparent diffusion coefficient. Iran J Radiol 13: e33133, 2016.

20. Shin HJ, Kim SH, Lee HJ, Gong G, Baek S, Chae EY, Choi WJ, Cha JH and Kim HH: Tumor apparent diffusion coefficient as an imaging biomarker to predict tumor aggressiveness in patients with estrogen-receptor-positive breast cancer. NMR Biomed 29: 1070-1078, 2016.

21. Hirano M, Satake H, Ishigaki S, Ikeda M, Kawai H and Naganawa S: Diffusion-weighted imaging of breast masses: Comparison of diagnostic performance using various apparent diffusion coefficient parameters. AJR Am J Roentgenol 198: 717-722, 2012.

22. Elston CW: Classification and grading of invasive breast carcinoma. Verh Dtsch Ges Pathol 89: 35-44, 2005.

23. Bellip P, Costantini M, Bufi E, Magistrelli A, La Torre G and Bonomo L: Diffusion-weighted imaging in breast lesion evaluation. Radiol Med 115: 51-69, 2010 (In English, Italian). 
24. Woodhams R, Matsunaga K, Kan S, Hata H, Ozaki M, Iwabuchi K, Kuranami M, Watanabe M and Hayakawa K: ADC mapping of benign and malignant breast tumors. Magn Reson Med Sci 4: 35-42, 2005.

25. Mori N, Ota H, Mugikura S, Takasawa C, Tominaga J, Ishida T, Watanabe M, Takase K and Takahashi S: Detection of invasive components in cases of breast ductal carcinoma in situ on biopsy by using apparent diffusion coefficient MR parameters. Eur Radiol 23: 2705-2712, 2013.

26. Kato F, Kudo K, Yamashita H, Wang J, Hosoda M, Hatanaka KC, Mimura R, Oyama-Manabe N and Shirato H: Differences in morphological features and minimum apparent diffusion coefficient values among breast cancer subtypes using 3-tesla MRI. Eur J Radio 185: 96-102, 2016.
27. Byun BH, Noh WC, Lim I, Lee SS, Cho AR, Park JA, Kim KM, Kim HA, Kim EK, Kim BI, et al: A new method for apparent diffusion coefficient measurement using sequential ${ }^{18}$ F-FDG PET and MRI: Correlation with histological grade of invasive ductal carcinoma of the breast. Ann Nucl Med 27: 720-728, 2013.

28. Marini C, Iacconi C, Giannelli M, Cilotti A, Moretti M and Bartolozzi C: Quantitative diffusion-weighted MR imaging in the differential diagnosis of breast lesion. Eur Radiol 17: 2646-2655, 2007.

29. Tamura T, Murakami S, Naito K, Yamada T, Fujimoto T and Kikkawa T: Investigation of the optimal b-value to detect breast tumors with diffusion weighted imaging by 1.5-T MRI. Cancer Imaging 14: 11, 2014. 